\title{
Quantum Spin Hall Effect in Graphene
}

\author{
C.L. Kane and E.J. Mele \\ Dept. of Physics and Astronomy, University of Pennsylvania, Philadelphia, PA 19104
}

\begin{abstract}
We study the effects of spin orbit interactions on the low energy electronic structure of a single plane of graphene. We find that in an experimentally accessible low temperature regime the symmetry allowed spin orbit potential converts graphene from an ideal two dimensional semimetallic state to a quantum spin Hall insulator. This novel electronic state of matter is gapped in the bulk and supports the transport of spin and charge in gapless edge states that propagate at the sample boundaries. The edge states are non chiral, but they are insensitive to disorder because their directionality is correlated with spin. The spin and charge conductances in these edge states are calculated and the effects of temperature, chemical potential, Rashba coupling, disorder and symmetry breaking fields are discussed.
\end{abstract}

PACS numbers: 73.43.-f, 72.25.Hg, 73.61.Wp, 85.75.-d

The generation of spin currents solid state systems has been a focus of intense recent interest. It has been argued that in doped semiconductors the spin orbit (SO) interaction leads to a spin-Hall effect 1, 2], in which a spin current flows perpendicular to an applied electric field. The spin Hall effect has been observed in GaAs 3, 4]. Murakami et al. [5] have identified a class of cubic materials that are insulators, but nonetheless exhibit a finite spin Hall conductivity. Such a "spin Hall insulator" would be of intrinsic interest, since it would allow for spin currents to be generated without dissipation.

In this paper we show that at sufficiently low energy a single plane of graphene exhibits a quantum spin-Hall (QSH) effect with an energy gap that is generated by the SO interaction. Our motivation is twofold. First, Novoselov et al. [6] have recently reported progress in the preparation of single layer graphene films. These films exhibit the expected ambipolar behavior when gated and have mobilities up to $10^{4} \mathrm{~cm}^{2} / \mathrm{Vs}$. Thus, the detailed experimental study of graphene now appears feasible. We believe the QSH effect in graphene is observable below a low but experimentally accessible temperature. Secondly, we will show the QSH effect in graphene is different from the spin hall effects studied for three dimensional cubic systems in Ref. 5 because it leads to a phase which is topologically distinct from a band insulator. The QSH effect in graphene resembles the charge quantum Hall effect, and we will show that spin and charge currents can be transported in gapless edge states. As a model system, graphene thus identifies a new class of spin Hall insulator. It may provide a starting point for the search for other spin-Hall insulators in two dimensional or in layered materials with stronger SO interaction.

SO effects in graphite have been known for over 40 years 7], and play a role in the formation of minority hole pockets in the graphite Fermi surface [8]. However, these effects have largely been ignored because they are predicted to be quite small and they are overwhelmed by the larger effect of coupling between the graphene planes. Unlike graphite which has a finite Fermi surface, however, graphene is in a critical electronic state which can be strongly affected by small perturbations at low energy.

Graphene consists of a honeycomb lattice of carbon atoms with two sublattices. The states near the Fermi energy are $\pi$ orbitals residing near the $K$ and $K^{\prime}$ points at opposite corners of the hexagonal Brillouin zone. An effective mass model can be developed [9] by writing the low energy electronic wavefunctions as

$$
\Psi(\mathbf{r})=\left[\left(u_{A K}, u_{B K}\right),\left(u_{A K^{\prime}}, u_{B K^{\prime}}\right)\right] \cdot \psi(\mathbf{r})
$$

where $u_{(A, B)\left(K, K^{\prime}\right)}(\mathbf{r})$ describe basis states at momentum $k=K, K^{\prime}$ centered on atoms of the $A, B$ sublattice. $\psi(\mathbf{r})$ is a four component slowly varying envelope function. The effective mass Hamiltonian then takes the form,

$$
\mathcal{H}_{0}=-i \hbar v_{F} \psi^{\dagger}\left(\sigma_{x} \tau_{z} \partial_{x}+\sigma_{y} \partial_{y}\right) \psi
$$

Here $\vec{\sigma}$ and $\vec{\tau}$ are Pauli matrices with $\sigma_{z}= \pm 1$ describing states on the $A(B)$ sublattice and $\tau_{z}= \pm 1$ describing states at the $K\left(K^{\prime}\right)$ points. This Hamiltonian describes gapless states with $E(\mathbf{q})= \pm v_{F}|\mathbf{q}|$. Without spin, the degeneracy at $\mathbf{q}=0$ is protected by symmetry. The only possible terms that could be added to open a gap are proportional to $\sigma_{z}$ or $\sigma_{z} \tau_{z}$. The $\sigma_{z}$ term, which corresponds to a staggered sublattice potential is odd under parity (which interchanges the $\mathrm{A}$ and $\mathrm{B}$ sublattices). The $\sigma_{z} \tau_{z}$ term is even under parity, but odd under time reversal (which interchanges $K$ and $K^{\prime}$ ).

The SO interaction allows for a new term, which will be the focus of this paper:

$$
\mathcal{H}_{S O}=\Delta_{s o} \psi^{\dagger} \sigma_{z} \tau_{z} s_{z} \psi
$$

Here $s_{z}$ is a Pauli matrix representing the electron's spin. This term respects all of the symmetries of graphene, and will be present. Below we will explicitly construct this term from the microscopic SO interaction and estimate its magnitude. If the mirror symmetry about the plane is preserved then this is the only allowed spin dependent term at $\mathbf{q}=0$. If the mirror symmetry is broken (either by a perpendicular electric field or by interaction with a 
substrate) then a Rashba term [10] of the form $(\mathbf{s} \times \mathbf{p}) \cdot \hat{z}$ is allowed,

$$
\mathcal{H}_{R}=\lambda_{R} \psi^{\dagger}\left(\sigma_{x} \tau_{z} s_{y}-\sigma_{y} s_{x}\right) \psi
$$

For $\lambda_{R}=0, \Delta_{s o}$ leads to an energy gap $2 \Delta_{s o}$ with $E(\mathbf{q})= \pm \sqrt{\left(\hbar v_{F} q\right)^{2}+\Delta_{s o}^{2}}$. For $0<\lambda_{R}<\Delta_{s o}$ the energy gap $2\left(\Delta_{s o}-\lambda_{R}\right)$ remains finite. For $\lambda_{R}>\Delta_{s o}$ the gap closes, and the electronic structure is that of a zero gap semiconductor with quadradically dispersing bands. In the following we will assume that $\lambda_{R}<\Delta_{s o}$ and analyze the properties of the resulting gapped phase. This assumption is justified by numerical estimates given at the end of the paper.

The gap generated by $\sigma_{z} \tau_{z} s_{z}$ is different from the gap that would be generated by the staggered sublattice potentials, $\sigma_{z}$ or $\sigma_{z} s_{z}$. The ground states in the presence of the latter terms are adiabatically connected to simple insulating phases at strong coupling where the two sublattices are decoupled. In contrast, the gap parameter $\sigma_{z} \tau_{z} s_{z}$ produces gaps with opposite signs at the $K$ and $K^{\prime}$ points. This has no simple strong coupling limit. To connect smoothly between the states generated by $\sigma_{z}$ and $\sigma_{z} \tau_{z} s_{z}$ one must pass through a critical point where the gap vanishes, separating ground states with distinct topological orders.

The interaction (3) is related to a model introduced by Haldane [11] as a realization of the parity anomaly in $(2+1)$ dimensional relativistic field theory. Taken separately, the Hamiltonians for the $s_{z}= \pm 1$ spins violate time reversal symmetry and are equivalent to Haldane's model for spinless electrons, which could be realized by introducing a periodic magnetic field with no net flux. As Haldane showed, this gives rise to a $\sigma_{z} \tau_{z}$ gap, which has opposite signs at the $K$ and $K^{\prime}$ points. At temperatures well below the energy gap this leads to a quantized Hall conductance $\sigma_{x y}= \pm e^{2} / h$. This Hall conductance computed by the Kubo formula can be interpreted as the topological Chern number induced by the Berry's curvature in momentum space 12, 13]. Since the signs of the gaps in (3) are opposite for opposite spins, an electric field will induce opposite currents for the opposite spins, leading to a spin current $\mathbf{J}_{s}=(\hbar / 2 e)\left(\mathbf{J}_{\uparrow}-\mathbf{J}_{\downarrow}\right)$ characterized by a quantized spin Hall conductivity

$$
\sigma_{x y}^{s}=\frac{e}{2 \pi} .
$$

Since spin currents do not couple to experimental probes it is difficult to directly measure (5). Moreover, the conservation of $s_{z}$ will be violated by the Rashba term (4) as well as terms which couple the $\pi$ and $\sigma$ orbitals. Nonetheless, Murakami et al. 14 have defined a conserved spin $s_{z(c)}$, allowing $\sigma_{x y}^{s}$ to be computed via the Kubo formula. We find that $\sigma_{x y}^{s}$ computed in this way is not quantized when $\lambda_{R} \neq 0$, though the correction to (5) is small due to carbon's weak SO interaction.
In the quantum Hall effect the bulk topological order requires the presence of gapless edge states. We now show that gapless edge states are also present in graphene. We will begin by establishing the edge states for $\lambda_{R}=0$. We will then argue that the gapless edge states persist even when $\lambda_{R} \neq 0$, and that they are robust against weak electron electron interactions and disorder. Thus, in spite of the violation of (5) the gapless edge states characterize a state which is distinct from an ordinary insulator. This QSH state is different from the insulators discussed in Ref. [5, which do not have edge states. It is also distinct from the spin Hall effect in doped GaAs, which does not have an energy gap.

For $\lambda_{R}=0$, the Hamiltonian $(2,3)$ conserves $s_{z}$, and the gapless edge states follow from Laughlin's argument 15. Consider a large cylinder (larger than $\left.\hbar v_{F} / \Delta_{s o}\right)$ and adiabatically insert a quantum $\phi=h / e$ of magnetic flux quantum down the cylinder (slower than $\left.\Delta_{s o} / \hbar\right)$. The resulting azimuthal Faraday electric field induces a spin current such that spin $\hbar$ is transported from one end of the cylinder to the other. Since an adiabatic change in the magnetic field cannot excite a particle across the energy gap $\Delta_{s o}$ it follows that there must be gapless states at each end to accommodate the extra spin.

An explicit description of the edge states requires a model that gives the energy bands throughout the entire Brillouin zone. Following Haldane 11, we introduce a second neighbor tight binding model,

$$
\mathcal{H}=\sum_{\langle i j\rangle \alpha} t c_{i \alpha}^{\dagger} c_{j \alpha}+\sum_{\langle\langle i j\rangle\rangle \alpha \beta} i t_{2} \nu_{i j} s_{\alpha \beta}^{z} c_{i \alpha}^{\dagger} c_{j \beta} .
$$

The first term is the usual nearest neighbor hopping term. The second term connects second neighbors with a spin dependent amplitude. $\nu_{i j}=-\nu_{j i}= \pm 1$, depending on the orientation of the two nearest neighbor bonds $\mathbf{d}_{1}$ and $\mathbf{d}_{2}$ the electron traverses in going from site $j$ to $i . \nu_{i j}=$ \pm 1 if the electron makes a left (right) turn to get to the second bond. The spin dependent term can be written in a coordinate independent representation as $i\left(\mathbf{d}_{1} \times \mathbf{d}_{2}\right) \cdot \mathbf{s}$. At low energy (6) reduces to $(2,3)$ with $\Delta_{s o}=3 \sqrt{3} t_{2}$.

The edge states can be seen by solving (7) in a strip geometry. Fig. 1 shows the one dimensional energy bands for a strip where the edges are along the zig-zag direction in the graphene plane. The bulk bandgaps at the one dimensional projections of the $K$ and $K^{\prime}$ points are clearly seen. In addition two bands traverse the gap, connecting the $K$ and $K^{\prime}$ points. These bands are localized at the edges of the strip, and each band has degenerate copies for each edge. The edge states are not chiral since each edge has states which propagate in both directions. However, as illustrated in Fig. 2 the edge states are "spin filtered" in the sense that electrons with opposite spin propagate in opposite directions. Similar edge states occur for armchair edges, though in that case the 1D projections of $K$ and $K^{\prime}$ are both at $k=0$. It is interesting to note that for zig-zag edges the edge states persist for $\Delta_{\text {so }} \rightarrow 0$, 


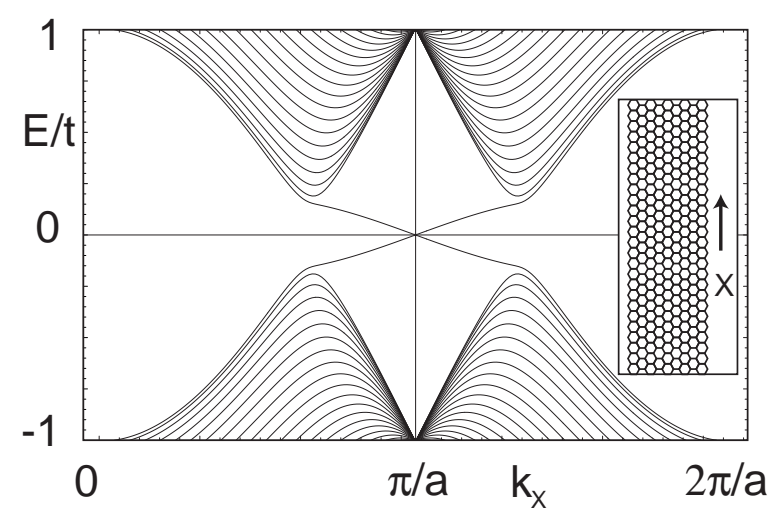

FIG. 1: (a) One dimensional energy bands for a strip of graphene (shown in inset) modeled by (7) with $t_{2} / t=.03$. The bands crossing the gap are spin filtered edge states.

where they become perfectly flat 16 . This leads to an enhanced density of states at the Fermi energy associated with zig-zag edges. This has been recently seen in scanning tunneling spectroscopy of graphite surfaces [17].

We have also considered a nearest neighbor Rashba term, of the form $i \hat{z} \cdot\left(\mathbf{s}_{\alpha \beta} \times \mathbf{d}\right) c_{i \alpha}^{\dagger} c_{j \beta}$. This violates the conservation of $s_{z}$, so that the Laughlin argument no longer applies. Nonetheless, we find that the gapless edge states remain, provided $\lambda_{R}<\Delta_{s o}$, so that the bulk bandgap remains intact. The crossing of the edge states at the Brillouin zone boundary $k_{x}=\pi / a$ in Fig. 1 (or at $k=0$ for the armchair edge) is protected by time reversal symmetry. The two states at $k_{x}=\pi / a$ form a Kramers doublet whose degeneracy cannot be lifted by any time reversal symmetric perturbation. Moreover, the degenerate states at $k_{x}=\pi / a \pm q$ are a Kramers doublet. This means that elastic backscattering from a random potential is forbidden. More generally, scattering from a region of disorder can be characterized by a $2 \times 2$ unitary Smatrix which relates the incoming and outgoing states: $\Phi_{\text {out }}=S \Phi_{\text {in }}$, where $\Phi$ is a two component spinor consisting of the left and right moving edge states $\phi_{L \uparrow}, \phi_{R \downarrow}$. Under time reversal $\Phi_{\text {in,out }} \rightarrow s_{y} \Phi_{\text {out,in }}^{*}$. Time reversal symmetry therefore imposes the constraint $S=s_{y} S^{T} s_{y}$, which rules out any off diagonal elements.

Electron interactions can lead to backscattering. For instance, the term $u \psi_{L \uparrow}^{\dagger} \partial_{x} \psi_{L \uparrow}^{\dagger} \psi_{R \downarrow} \partial_{x} \psi_{R \downarrow}$, does not violate time reversal, and will be present in an interacting Hamiltonian. For weak interactions this term is irrelevant under the renormalization group, since its scaling dimension is $\Delta=4$. It thus will not lead to an energy gap or to localization. Nonetheless, it allows inelastic backscattering. To leading order in $u$ it gives a finite conductivity of the edge states, which diverges at low temperature as $u^{-2} T^{3-2 \Delta}[18]$. Since elastic backscattering is prevented by time reversal there are no relevant backscattering processes for weak interactions. This stability against interactions and disorder distinguishes the

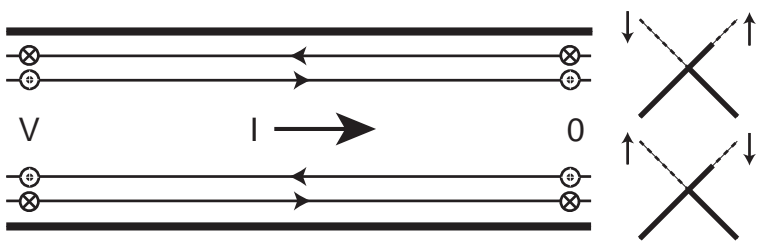

(a)

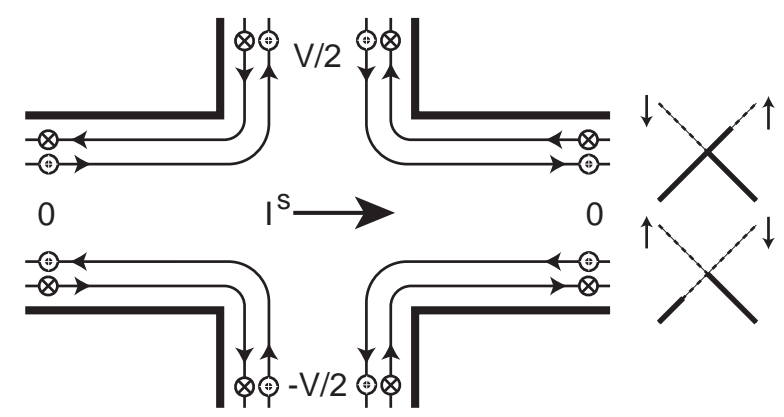

(b)

FIG. 2: Schematic diagrams showing (a) two terminal and (b) four terminal measurement geometries. In (a) a charge current $I=\left(2 e^{2} / h\right) V$ flows into the right lead. In (b) a spin current $I^{s}=(e / 4 \pi) V$ flows into the right lead. The diagrams to the right indicate the population of the edge states.

spin filtered edge states from ordinary one dimensional wires, which are localized by weak disorder.

A parallel magnetic field $H_{\|}$breaks time reversal and leads to an avoided crossing of the edge states. $H_{\|}$also reduces the symmetry, allowing terms in the Hamiltonian which provide a continuously gapped path connecting the states generated by $\sigma_{z} \tau_{z} s_{z}$ and $\sigma_{z}$. Thus in addition to gapping the edge states $H_{\|}$eliminates the topological distinction between the QSH phase and a simple insulator.

The spin filtered edge states have important consequences for both the transport of charge and spin. In the limit of low temperature we may ignore the inelastic backscattering processes, and describe the ballistic transport in the edge states within a Landauer-Büttiker 19 framework. For a two terminal geometry (Fig. 2a), we predict a ballistic two terminal charge conductance $G=2 e^{2} / h$. For the spin filtered edge states the edge current density is related to the spin density, since both depend on $n_{R \uparrow}-n_{L \downarrow}$. Thus the charge current is accompanied by spin accumulation at the edges. The interplay between charge and spin can be probed in a multiterminal device. Define the multiterminal spin conductance by $I_{i}^{s}=\sum_{j} G_{i j}^{s} V_{j}$. Time reversal symmetry requires $G_{j i}^{s}=-G_{i j}^{s}$, and from Fig. $2 \mathrm{~b}$ it is clear that $G_{i j}^{s}= \pm e / 4 \pi$ for adjacent contacts $i$ and $j$. In the four terminal geometry of Fig. 2b a spin current $I^{s}=e V / 4 \pi$ flows into the right contact. This geometry can also be used to measure a spin current. A spin current incident from the left (injected, for instance with a ferromagnetic contact) will be split, with the up (down) spins trans- 


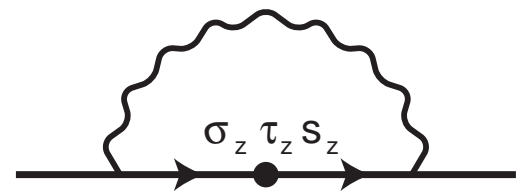

FIG. 3: Feynman diagram describing the renormalization of the SO potential by the Coulomb interaction. The solid line represents the electron propagator and the wavy line is the Coulomb interaction.

ported to the top (bottom contacts), generating a measurable spin-Hall voltage.

The magnitude of $\Delta_{\text {so }}$ may be estimated by treating the microsopic SO interaction

$$
V_{S O}=\frac{\hbar}{4 m^{2} c^{2}} \mathbf{s} \cdot(\nabla V \times \mathbf{p})
$$

in first order degenerate perturbation theory. We thus evaluate the expectation value of (8) in the basis of states given in (1) treating $\psi(\mathbf{r})$ as a constant. A full evaluation depends on the detailed form of the Bloch functions. However a simple estimate can be made in the "first star" approximation: $u_{\left(K, K^{\prime}\right),(A, B)}(\mathbf{r})=\sum_{p} \exp \left[i \mathbf{K}_{p}\right.$. $(\mathbf{r}-\mathbf{d})] / \sqrt{3}$. Here $\mathbf{K}_{p}$ are the crystal momenta at the three corners of the Brillouin zone equivalent to $K$ or $K^{\prime}$, and $\mathbf{d}$ is the a basis vector from a hexagon center to an A or B sublattice site. We find that the matrix elements have precisely the structure (3), and using the Coulomb interaction $V(r)=e^{2} / r$ we estimate $2 \Delta_{s o}=4 \pi^{2} e^{2} \hbar^{2} /\left(3 m^{2} c^{2} a^{3}\right) \sim 2.4^{\circ} K$. This is a crude estimate, but it is comparable to the SO splittings quoted in the graphite literature 8 .

The Rashba interaction due to a perpendicular electric field $E_{z}$ may be estimated as $\lambda_{R}=\hbar v_{F} e E_{z} /\left(4 m c^{2}\right)$. For $E_{z} \sim 50 \mathrm{~V} / 300 \mathrm{~nm}[\underline{3}]$ this gives $\lambda_{R} \sim .5 \mathrm{mK}$. This is smaller than $\Delta_{s o}$ because $E_{z}$ is weaker than the atomic scale field. The Rashba term due to interaction with a substrate is more difficult to estimate, though since it is presumably a weak Van der Waals interaction, this too can be expected to be smaller than $\Delta_{s o}$.

This estimate of $\Delta_{\text {so }}$ ignores the effect of electron electron interactions. The long range Coulomb interaction may substantially increase the energy gap. To leading order the SO potential is renormalized by the diagram shown in Fig. 3, which physically represents the interaction of electrons with the exchange potential induced by $\Delta_{s o}$. This is similar in spirit to the gap renormalizations in 1D Luttinger liquids and leads to a logarithmically divergent correction to $\Delta_{s o}$. The divergence is due to the long range $1 / r$ Coulomb interaction, which persists in graphene even accounting for screening [20]. The divergent corrections to $\Delta_{s o}$ as well as similar corrections to $\hbar v_{F}$ can be summed using the renormalization group (RG) 20]. Introducing the dimensionless Coulomb interaction $g=e^{2} / \hbar v_{F}$ we integrate out the high energy degrees of freedom with energy between $\Lambda$ and $\Lambda e^{-\ell}$. To leading order in $g$ the RG flow equations are

$$
d g / d \ell=-g^{2} / 4 ; \quad d \Delta_{s o} / d \ell=g \Delta_{s o} / 2 .
$$

These equations can be integrated, and at energy scale $\varepsilon$, $\Delta_{\text {so }}(\varepsilon)=\Delta_{\text {so }}^{0}\left[1+\left(g^{0} / 4\right) \log \left(\Lambda^{0} / \varepsilon\right)\right]^{2}$. Here $g^{0}$ and $\Delta_{\text {so }}^{0}$ are the interactions at cutoff scale $\Lambda^{0}$. The renormalized gap is determined by $\Delta_{s o}^{R} \sim \Delta_{s o}\left(\Delta_{s o}^{R}\right)$. Using an effective interaction $g^{0}=.74[21]$ and $\Lambda^{0} \sim 2 \mathrm{eV}$ this leads to $2 \Delta_{\text {so }}^{R} \sim 15^{\circ} \mathrm{K}$.

In summary, we have shown that the ground state of a single plane of graphene exhibits a QSH effect, and has a non trivial topological order that is robust against small perturbations. The QSH phase should be observable by studying low temperature charge transport and spin injection in samples of graphene with sufficient size and purity to allow the bulk energy gap to manifest itself. It would also be of interest to find other materials with stronger SO coupling which exhibit this effect, as well as possible three dimensional generalizations.

We thank J. Kikkawa and S. Murakami for helpful discussions. This work was supported by the NSF under MRSEC grand DMR-00-79909 and the DOE under grant DE-FG02-ER-0145118.

[1] S. Murakami, N. Nagaosa and S.C. Zhang, Science 301, 1348 (2003).

[2] J. Sinova et al., Phys. Rev. Lett. 92, 126603 (2004).

[3] Y.K. Kato et al., Science 306, 1910 (2004).

[4] J. Wunderlich et al., cond-mat/0410295 (2004).

[5] S. Murakami, N. Nagaosa and S.C. Zhang, Phys. Rev. Lett. 93, 156804 (2004).

[6] K.S. Novoselov, et al., Science 306, 666 (2004).

[7] G. Dresselhaus and M.S. Dresselhaus, Phys. Rev. 140, A401 (1965).

[8] N.B. Brandt, S.M. Chudinov and Y.G. Ponomarav, Semimetals 1. Graphite and its compounds (North Holland, 1988).

[9] D.P. DiVincenzo and E.J. Mele, Phys. Rev. B 29, 1685 (1984).

[10] Y.A. Bychkov and E.I. Rashba, J. Phys. C 17, 6039 (1984).

[11] F.D.M. Haldane, Phys. Rev. Lett. 61, 2015 (1988).

[12] D.J. Thouless, M. Kohmoto, M.P. Nightingale and M. den Nijs, Phys. Rev. Lett 49, 405 (1982).

[13] F.D.M. Haldane, Phys. Rev. Lett. 93, 206602(2004).

[14] S. Murakami, N. Nagaosa and S.C. Zhang, Phys. Rev. B 69, 235206 (2004).

[15] R.B. Laughlin, Phys. Rev. B 23, R5632 (1981).

[16] M. Fujita et al. J. Phys. Soc. Jpn. 65, 1920 (1996).

[17] Y. Niimi, et al., Phys. Rev. B 70, 214430 (2004).

[18] T. Giamarchi and H. Schulz, Phys. Rev. B 37, 325 (1988).

[19] M. Büttiker, Phys. Rev. B 38, 9375 (1988).

[20] J. Gonzalez, F. Guinea and M.A.H. Vozmediano, Phys. Rev. B 59, R2474 (1999).

[21] C.L. Kane and E.J. Mele, Phys. Rev. Lett. 93, 197402 (2004). 